Methods We searched the electronic bibliographic databases, including Cochrane Central Register of Controlled Trials (Issue 1, 2010), PubMed (1994 2010.5). EMBASE (1994 2010.5), CNKI (1994 2010.5), VIP (1994 2010.5), Wanfang database (1994 2010.5) to assemble the randomised controlled trials (RCTs) of RVS Pacing compared with right ventricular apical (RVA) pacing. Two reviewers evaluated the quality of included studies based on the Handbook 5.0.2 and extracted data independently. Meta-analysis was performed by RevMan 5.0 software.

Results 35 RCTs involving 2054 patients were included. The results of meta-analysis showed: compared with the RVA pacing, RVS pacing could significantly reduce the ORS wave duration $(\mathrm{MD}=-0.05,95 \% \mathrm{CI}-0.07$ to -0.02$)$, significantly increase the left ventricular ejection fraction of 3 months and 18 months after operation ( $\mathrm{MD}=7.10,95 \% \mathrm{CI} 3.03$ to 11.17$) ;(\mathrm{MD}=7.44,95 \% \mathrm{CI}$ 5.46 to 9.42 ). 3 months later, there was no significant difference between the two groups with regard to pacing threshold $(\mathrm{MD}=-13.88$, 95\% CI -29.75 to 2.00$)$, Compared with RVA, RVS was associated with a significant reduction in threshold perception current $(\mathrm{MD}=-0.73,95 \% \mathrm{CI}-29.75$ to -0.12$)$ and impedance $(\mathrm{MD}=-75.12,95 \% \mathrm{CI}-35.53$ to -14.71$)$.

Conclusion RVS pacing can give patients a good physiological state which is more consistent with biventricular electric conduction, and lead to haemodynamic improvement. RVS pacing might be expected to become a preferred site of ventricular pacing.

\section{E0193 A CRITICAL ROLE OF CKIT IN CXCR4-MEDIATED PROGENITOR CELL NICHE MAINTENANCE AND MOBILISATION}

doi:10.1136/hrt.2010.208967.193

Cheng Min, Zeng Qiutang, Losordo Douglas. Wuhan Union Hospital

Therapeutic mobilisation of bone marrow progenitor cells (BM PCs) is a novel strategy for cardiovascular repair. Both CXCR4 antagonism and c-kit blockade can rapidly and potently mobilise BM PCs; however, the functional interaction between CXCR4 and c-kit remains unclear. We treated c-kit-deficient (c-kit W/W-V $)$ and wildtype (WT) mice with CXCR4 antagonist AMD3100 and evaluated $\mathrm{PCs}$ in the peripheral blood (PB) with a colony-forming assay. AMD3100 treatment for $2 \mathrm{~h}$ dramatically increased the number of $\mathrm{PCs}$ in the PB in WT mice but not in c-kit ${ }^{\mathrm{W}} / \mathrm{W}-\mathrm{V}$ mice (c-kit ${ }^{\mathrm{W} / \mathrm{W}-\mathrm{V}}$ vs WT: 30 vs 194 colonies/ml blood, $\mathrm{p}<0.01$ ). To confirm that $\mathrm{c}-\mathrm{kit}$ deficiency impairs BM PC mobilisation by AMD3100, we developed an in vivo BM niche clearance/occupation assay. The c-kit ${ }^{\mathrm{W}} / \mathrm{W}-\mathrm{V}$ and WT mice were firstly treated with AMD3100 to remove PCs from the niche, and $2 \mathrm{~h}$ later, transplanted with eGFP transgenic BM cells to competitively occupy the niche. After $3 \mathrm{~h}, \mathrm{BM}$ cells were isolated and analysed by FACS. Despite the total donor-derived (eGFP+) cells were similar between WT and c-kit ${ }^{\mathrm{W} / \mathrm{W}-\mathrm{V}}$ recipients, the donorderived CXCR4-expressing PCs, including $\mathrm{eGFP}^{+} \mathrm{CXCR} 4^{+} \mathrm{Lin}^{-}$,

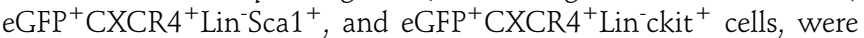
much fewer in the c-kit ${ }^{\mathrm{W} / \mathrm{W}-\mathrm{V}}$ mice (c-kit ${ }^{\mathrm{W} / \mathrm{W}-\mathrm{V}}$ vs WT: $19.7 \%$ vs $30.6 \%, \mathrm{p}<0.01 ; 20.3 \%$ vs $29.1 \%, \mathrm{p}<0.05$; and $6.7 \%$ vs $17.9 \%$, $\mathrm{p}<0.001$, respectively), indicating that c-kit deficiency specifically reduced the capacity of AMD3100 to clear the CXCR4 ${ }^{+}$PC niche. To better understand the mechanisms, we designed an ex vivo adhesion assay. Mouse BM mononuclear cells were isolated and applied onto plates pre-coated with BM stromal protein VCAM-1, followed by addition of AMD3100. The adhesion of BM cells to VCAM-1 resulted in marked c-kit phosphorylation. Interestingly, AMD3100 significantly attenuated the c-kit phosphorylation. In vivo, AMD3100 treatment for 15 min significantly reduced the level of phospho-c-kit in the BM as assessed by Western blotting of the $\mathrm{BM}$ lysates. Consistently, immunofluorescence staining of BM niche demonstrated a significantly lower ratio of phospho-ckit ${ }^{+} /$total $\mathrm{ckit}^{+} \mathrm{PCs}$ in AMD3100-treated mice as compared to PBS-treated mice. We conclude that c-kit plays a critical role in CXCR4-mediated BM PC niche maintenance and mobilisation.

\section{e0194 SRC FAMILY KINASE SFK IS ESSENTIAL FOR RECRUITMENT OF BONEMARROW PROGENITOR CELLS TO THE ISCHAEMIC MYOCARDIUM}

doi:10.1136/hrt.2010.208967.194

Cheng Min, Zeng Qiutang, Losordo Douglas. Wuhan Union Hospital, Wuhan, China

Background The G protein-coupled receptor CXCR4 and its ligand stromal-cell derived factor 1 (SDF-1) play an important role in directing progenitor cells (PC) homing to ischaemic tissue. The Src family protein kinases (SFK) can be activated by, and serve as effectors of, G proteins. However, whether SFK play a role in SDF-1/ CXCR4-mediated PC homing is unknown.

Methods and results To investigate whether SDF-1-CXCR4 signalling activates SFK, we isolated mouse bone marrow mononuclear cells (BM MNCs) and applied onto VCAM1-coated plates, followed by addition of CXCR4 agonist SDF-1 and/or antagonist AMD3100. SDF-1 rapidly (in $2 \mathrm{~min}$ ) and dose-dependently increased phosphorylation (activation) of Lyn, a major SFK in the BM; AMD3100 attenuated the SDF-1-induced Lyn phosphorylation. Notably, SDF1 treatment did not increase Lyn phosphorylation in the BM MNCs isolated from $\mathrm{Mx} 1-\mathrm{cre}^{+} \mathrm{CXCR} 4^{\mathrm{fl} / \mathrm{fl}}$ mice in which the CXCR4 gene had been deleted. To investigate whether SFK play a role in SDF-1/ CXCR4-mediated chemotaxis, we performed Boyden chamber migration assay; SU6656, a SFK inhibitor, significantly inhibited $\mathrm{BM}-\mathrm{MNC}$ migration towards SDF-1 $(\mathrm{p}<0.001, \mathrm{n}=4)$. To investigate whether SFK play a role in SDF-1/CXCR4-mediated BM-MNC homing to ischaemic heart tissue, we isolated BM MNCs from CXCR $4_{\text {BAC }}$ :eGFP transgenic mice and injected $1 \times 10^{6}$ cells into WT

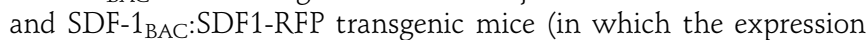
of SDF1-RFP fusion protein is driven by the SDF-1 genomic regulatory sequence and the level of total SDF-1 protein is doubled) that had undergone surgical myocardial infarction $8 \mathrm{~h}$ earlier. Some recipient mice also received two i.p. injections of SU6656 $(6 \mathrm{mg} / \mathrm{kg})$ at the time of cell injection and again $4 \mathrm{~h}$ later. We found a significantly greater amount of eGFP + cells (1.6-fold, $\mathrm{p}<0.01, \mathrm{n}=5$ ) and eGFP+c-kit + cells $(1.9$-fold, $\mathrm{p}<0.01, \mathrm{n}=5)$ recruited in the infarct border area of the SDF- $1_{\mathrm{BAC}}$ : SDF1-RFP recipients than in WT recipients. SU6656 treatments significantly reduced the amount of eGFP+ cells and eGFP+c-kit + cells $(p<0.01, n=5)$ in both WT and SDF- $1_{\text {BAC: }}$ RFP recipients and abrogate the difference between the two groups.

Conclusions SFK play a critical role in SDF-1/CXCR4-mediated BM $\mathrm{PC}$ homing to the ischaemic cardiac tissue thus may provide a target for modulation of tissue repair.

\section{e0195 RESEARCH ON THE EVALUATION OF OCT ON ATHEROSCLEROTIC PLAOUES OF CAROTID ARTERY IN RABBITS WITH INSULIN RESISTANCE}

doi:10.1136/hrt.2010.208967.195

Zhiping Shen, Zhiping Shen. Tianjin People's Hospital, Tianjin, China

Objective The purpose was to analyse the feasibility and repeatability of OCT to evaluate atherosclerotic plaque diagnosed by pathologic examination of common carotid artery in rabbits with insulin resistance.

Methods There were 26 male China White rabbits. 20 rabbits were made atherosclerotic with a high-cholesterol diet after injury of the 
left carotid artery endothelium. Another 6 rabbits were fed common diet, no injury. At the beginning and feeding for 12 weeks, TG, TC, HDL, LDL, FBG, fasting insulin and insulin sensitivity index were tested, analysis the correlation between HOMA-IR and lipid. After 12 weeks, OCT images of the left carotid artery were performed. After OCT, specimens were taken. Histology embedded in paraffin and $\mathrm{HE}$ stained was performed on arterial regions that was showed plaques in the OCT scan. OCT results were compared with pathological results.

Results 4 rabbits died, no death in normal. Feeding high fat diet for 12 weeks, their weight, TC, TG, LDL-C, HDL-C were significantly higher than normal $(p<0.01)$. Though the difference of FBG was not statistically significant compared with normal $(p=0.423)$, FSI, ISI and HOMA-IR was statistically significantly different $(\mathrm{p}=0.001$, $0.000,0.000$ respectively). HOMA-IR and TG were positively correlated $(\mathrm{r}=0.52, \mathrm{p}=0.039)$, and TC, HDL, LDL no significant correlation $(p>0.05)$. OCT images of the left carotid artery in 22 rabbits were performed. 60 OCT images analysis after two experts' discussions were held. There were 50 lipid-rich plaques, seven fibrous plaques and three suspected as calcified plaques. In addition, four thrombosis and intima tear in two sites were found. The pathological section was made in corresponding sites to OCT, HE staining. Comparing with the pathology results, OCT had high sensitivity and specificity for atherosclerotic plaque, respectively 96\%, 89\%. Plaque burden, external elastic membrane area and lumens area between the two methods were not statistically different $(p>0.05)$.

Conclusion OCT imaging can clearly visualise different types of atherosclerotic plaques and provide detailed information on plaque characteristics. Comparing with histopathology, OCT had high sensitivity and specificity for characterising atherosclerotic plaque.

\section{e0196 TRB3'-SILENCE REMARKABLY ATTENUATES RENAL FIBROSIS IN DIABETIC RAT}

doi:10.1136/hrt.2010.208967.196

Ding Wenyuan, Ti Yun, Wang Zhihao. Qilu Hospital

Background Renal fibrosis is thought to be the common pathway in most cases of end-stage renal diseases. Recently, it is reported that tribble3 plays an important role in the progress of cardiac fibrosis in diabetes mellitus (DM). Therefore, TRB3 might participate in the pathogenesis of renal fibrosis of diabetes mellitus rat.

Methods 84 male Wistar rats were randomly divided into three groups: control group $(n=12)$, high-fat diet group (HF group, $n=36$ ), DM group $(n=36)$. The rats in DM group were injected with streptozotocin (STZ) after feeding with a high-fat diet for 4 weeks. The last two groups were re-divided into three subgroups according to injection with TRB3 siRNA adenovirus at week 17 whether or not (HF+Ad group; HF+Vector group; HF group; DM+Ad group; $\mathrm{DM}+$ Vector group; DM group; $\mathrm{n}=12$ per group). After rats sacrificed, renal tissue was removed and stained with $\mathrm{H} \& \mathrm{E}$ and masson's trichrosome staining.

Results Renal fibrosis was significantly increased in DM group compared to HF group and Control group $(4.1 \pm 0.87$ vs $0.9 \pm 0.13$ vs $0.53 \pm 0.08 ; p<0.05, p=0.017$, respectively). The severity of fibrosis was also significantly different between three subgroups in DM group, the same to HF group. In DM group, renal fibrosis obviously ameliorate in $\mathrm{DM}+\mathrm{Ad}$ group compared to $\mathrm{DM}+$ Vector group and DM group ( $1.4 \pm 0.24$ vs $7.1 \pm 0.8$ vs $3.7 \pm 0.8 ; \mathrm{p}<0.0001, \mathrm{p}<0.01$, respectively). In HF group, renal fibrosis also noticeably improvement in $\mathrm{HF}+\mathrm{Ad}$ group compared to HF+Vector group and HF group ( $1.3 \pm 0.24$ vs $3.0 \pm 0.8$ vs $0.9 \pm 0.13 ; p<0.05, p<0.05$, respectively).

Conclusion TRB3'silence remarkably attenuates renal fibrosis and gene interference shows beneficial effect in the development of diabetic nephropathy in diabetes mellitus.

\section{e0197 EFFECTS OF PRIOR DIFFERENT INTENSITIES EXERCISE ON INFARCT REGION FUNCTION AND ANGIOGENESIS OF LEFT VENTRICLE IN MYOCARDIAL INFARCTION RATS}

doi:10.1136/hrt.2010.208967.197

${ }^{1}$ Xiang-Yu Tang, ${ }^{2}$ Hua-Shan Hong, 'Liang-Long Chen, ${ }^{1}$ Xiao-Hong Lin, ${ }^{1} J u n-H u a$ Lin, 'Zhang Lin. 'Department of Cardiology of Union Hospital Affiliated to Fujian Medical University; ${ }^{2}$ Department of Geriatrics of Union Hospital Affiliated to Fujian Medical University, Fuzhou, Fujian, China, 350001

Objective Lifestyle interventions including exercise training have been shown to be a feasible option for the prevention and treatment of cardiovascular diseases. In this study, we investigated the effects of prior different intensities of exercise on infarct region and function and angiogenesis of the left ventricle (LV) in post-myocardial infarction (MI) rats, and further examined the expression of VEGF proteins in the left ventricle.

Methods Male Sprague-Dawley rats were randomly assigned to six groups. The exercised rats underwent a daily 60-min treadmill exercise, $5 \mathrm{~d} / \mathrm{wk}$, for $6 \mathrm{wk}$. Different treadmill speeds were carried out in the high intensity exercise group (HIE-MI group, $30 \mathrm{~m} / \mathrm{min}$ ), moderate intensity exercise groups (MIE-Sh and MIE-MI groups, $21 \mathrm{~m} / \mathrm{min}$ ), low intensity exercise group (LIE-MI group, $12 \mathrm{~m} / \mathrm{min}$ ), respectively, whereas the untrained rats (Sed-Sh and Sed-MI groups) remained sedentary. At $6 \mathrm{wk}, 24 \mathrm{~h}$ after the last treadmill exercise or the corresponding sedentary protocol, all rats underwent either acute MI (LIE-MI, MIE-MI, HIE-MI and Sed-MI groups) or sham MI operation (MIE-Sh and Sed-Sh groups). Then, all rats were sacrificed at $7 \mathrm{~d}$ after recovery. Echocardiographic and haemodynamic measurements were performed at the end of the experimental protocol, and hearts were sampled for histological and molecular analysis. The infarct regions were analysed by using Masson's trichrome staining; intramyocardial microvessels were detected by using Factor VIIIrelated antigen staining; and cardiac VEGF protein levels were determined by Western blotting analysis.

Results Compared with Sed-Sh group, MIE-Sh group ameliorated left ventricular function and increased microvessels intensity, though the difference was not significant. Compared with Sed-MI group, MIE-MI and HIE-MI groups significantly reduced left ventricular infarct size, improved haemodynamic parameters, increased fractional shortening, scar thickness and microvessel density, but LIE-MI rats only had slight, and non-significant effect on these parameters. In addition, the differences between MIE-MI and HIE-MI rats were significant in haemodynamic parameters and microvessel density. Interestingly, compared with Sed-MI group, the exercised hearts displayed higher levels of VEGF protein in MIE-MI and HIE-MI groups. However, there was no significant difference between MIE-MI and HIE-MI groups.

Conclusion Moderate intensity running exercise before acute MI improved LV function, reduced scar size, increased scar thickness and microvessel density in the post-MI rat. Additional higher intensity exercise could have little further effect. Low intensity exercise may be beneficial, but not sufficient to improve MI Moderate and high intensity running upregulated the expression of VEGF protein, and contributed to the increased microvessels, which may partly benefit cardiac function after MI.

\section{e0198 ACTIVITY OF CALCINEURIN NFAT SIGNALLING PATHWAY INVOLVING IN REMODELLING IN CTNI R146W MICE}

doi:10.1136/hrt.2010.208967.198

Zhu Shushu, Ma Jizheng, Chen Xiangjian, Wu Hengfang, Yang Di, Zhang Jinan. Nanjing Medical University, China

Objective To construct a transgenic model of HCM overexpressing $\mathrm{cTnI}^{\mathrm{R} 146 \mathrm{~W}}$, observe the pathological change of this animal, and 